\title{
eJRIEPS
}

Ejournal de la recherche sur l'intervention en éducation physique et sport

$47 \mid 2020$

Varia

Intervention en école primaire pour promouvoir l'activité physique et diminuer le temps sédentaire : bilan des expériences vécues par des enseignants

School-based intervention to promote physical activity and reduce sedentary time: a survey of the teachers' experiences

\section{Caroline Bernal}

\section{OpenEdition}

\section{Journals}

Édition électronique

URL : http://journals.openedition.org/ejrieps/5297

DOI : 10.4000/ejrieps.5297

ISSN : 2105-0821

Éditeur

ELLIADD

\section{Référence électronique}

Caroline Bernal, « Intervention en école primaire pour promouvoir l'activité physique et diminuer le temps sédentaire : bilan des expériences vécues par des enseignants », eJRIEPS [En ligne], 47 | 2020, mis en ligne le 01 juillet 2020, consulté le 21 novembre 2020. URL : http://journals.openedition.org/ ejrieps/5297 ; DOI : https://doi.org/10.4000/ejrieps.5297

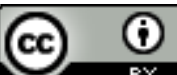

La revue eJRIEPS est mise à disposition selon les termes de la Creative Commons Attribution 4.0 International License. 


\section{Intervention en école primaire pour promouvoir l'activité physique et diminuer} le temps sédentaire : bilan des expériences vécues par des enseignants

Caroline Bernal ${ }^{*}$

*Université de Pau et des Pays de l'Adour, e2s UPPA, MEPS, Tarbes, France

\section{Résumé}

Face à l'augmentation de l'inactivité physique, une intervention ayant pour objectif de promouvoir l'activité physique et de diminuer le temps sédentaire des enfants a été mise en place successivement dans deux écoles primaires françaises situées en Réseau d'Education Prioritaire. Suite aux interventions, des données de questionnaires destinés aux enseignants ont permis de connaître leur investissement et le déroulement de l'intervention sur le terrain. Globalement, les enseignants ont adhéré à l'intervention en proposant en toute autonomie des pauses actives pour réduire le temps sédentaire ainsi que des ateliers d'activité physique supplémentaires. L'intervention a également permis d'agir positivement sur leur propre niveau d'activité physique. Ce partage d'expérience peut constituer une source d'informations pour les futurs chercheurs, intervenants et enseignants s'intéressant à la promotion des comportements de santé pendant l'enfance.

Mots clés : activité physique, sédentarité, intervention, école, enseignant

School-based intervention to promote physical activity and reduce sedentary time: a survey of the teachers' experiences

\section{Summary}

With the increase of physical inactivity levels, an intervention aimed at promoting physical activity and reducing children's sedentary time has been implemented in one french primary school and duplicated in a second primary school. Data from questionnaires addressed to the teachers following theses interventions provided a feedback on the progress of the interventions and on the experiences lived in the school. Teachers have globally adhered to the intervention by proposing independently sedentary breaks and additional physical activity workshops. The intervention also had 


\section{eJRIEPS 47 Juillet 2020}

a positive impact on their personal physical activity level. This shared experience from teachers can be useful for future researchers, practitioners and teachers who are interested in the promotion of health behaviours on childhood.

Key words: physical activity, sedentariness, intervention, school, teacher

\section{Introduction}

Dans les pays industrialisés tels que la France, le développement de l'ère industrielle a précipité le déclin de l'activité physique (AP) et a augmenté parallèlement le temps passé assis (Warburton \& Bredin, 2017). Les enfants d'aujourd'hui, nés dans l'Anthropocène, ont connu uniquement cette société hypermoderne qui a pour norme la réalisation d'activités en position sédentaire et qui considère la pratique d'AP comme complémentaire au mode de vie traditionnel (Cregan-Reid, 2019). Les comportements sédentaires sont inconscients et automatisés (Cheval, Radel, Neva, Boyd \& Swinnen, 2018). Ainsi, se faire accompagner par ses parents en voiture, prendre le bus ou un ascenseur, s'asseoir pendant des heures à l'école, constituent des comportements habituels. Tous ces temps sédentaires s'additionnent au temps consacré aux loisirs passifs, comme par exemple le temps passé face aux écrans, qui ont été démultipliés ces dernières décennies par les avancées technologiques (Cregan-Reid, 2019). Ainsi, en France, $72 \%$ des garçons et $82 \%$ des filles ne respectent pas les recommandations des 60 minutes d'AP par jour et le temps sédentaire (TS) est estimé à plus de 8 heures par jour pour chaque enfant (Rapport de Santé Publique France, 2015). De plus, pendant les années d'école primaire, entre 6 et 12 ans, l'AP décroît significativement (Cheval \& al., 2018). Ce fléchissement s'accélère ensuite tout au long de l'adolescence ainsi qu'à l'âge adulte. Or, l'inactivité physique et le cumul de TS entraînent de nombreuses répercussions négatives sur la santé physique, psychologique et sociale et sont considérés comme des problèmes de santé publique graves (Warburton \& Bredin, 2017). L'inactivité physique a notamment été identifiée comme étant la quatrième cause de mortalité au niveau mondial. Les problèmes de santé engendrés pendant l'enfance et l'adolescence ont des répercussions sur la future vie adulte de l'enfant (Jones, Hinkley, Okely \& Salmon, 2013), augmentant le risque de développer des pathologies cardiovasculaires. Face à ce constat, il est primordial d'agir pour modifier les comportements de santé des enfants. 


\section{Cadre théorique}

La prévalence de l'inactivité physique et de la sédentarité est plus élevée pour des enfants issus de milieux défavorisés (Cheval \& al., 2018). En effet, ces milieux de vie diminuent les possibilités de pratiquer une AP. Certains facteurs de stress environnementaux (trafic de drogue, violence dans le quartier) peuvent amener à réduire la pratique d'AP récréative dans le quartier (King, Stockols, Talen \& Killingsworth, 2002 ; Perkins, Meeks \& Taylor, 1992 ; Sanders-Phillips, 2000). De plus, les moyens logistiques, financiers et éducationnels des familles constituent également des barrières importantes à la pratique d'AP (King \& al., 2002). L'école permet de réaliser des activités de sensibilisation et d'éducation à la santé auprès de tous les enfants, quelle que soit leur situation familiale et financière. En effet, les comportements actifs doivent être revalorisés et être réappris dans ce contexte d'urgence sanitaire (World Health Organization, 2019). Le rôle du chercheur est de concevoir des interventions dans le domaine scolaire et d'évaluer leurs effets afin de promouvoir les comportements actifs des enfants tout au long de leur vie (Kriemler \& al., 2011).

II semble que les interventions en école primaire destinées à promouvoir l'AP et diminuer le TS qui sont les plus efficaces sont celles qui agissent sur une somme de facteurs qui influencent les enfants (Kriemler \& al., 2011). Le modèle socio-écologique regroupe l'ensemble de ces facteurs (Sallis, Owen et Fisher, 2008). L'enfant y est placé au centre avec ses caractéristiques personnelles. II est influencé par des facteurs interpersonnels situés au second niveau du modèle, tels que les parents et les enseignants. Ainsi, les études interventionnelles en école primaire proposent par exemple des ateliers de sensibilisation pour les parents et des formations pour les enseignants afin qu'ils puissent par la suite influencer positivement les enfants dans l'adoption de comportements sains. Le troisième niveau est composé de facteurs environnementaux et organisationnels. Par exemple, il est possible d'aménager le milieu scolaire en mettant à disposition du matériel sportif aux enfants pendant les récréations ainsi que proposer des créneaux d'AP supplémentaires à l'école. Enfin, au niveau le plus distal du modèle, les politiques publiques peuvent également influencer les enfants. Par exemple, les politiques de la ville et des régions sont en mesure de décider d'implanter des parcs ou de favoriser les activités sportives dans la commune. Afin d'augmenter la motivation intrinsèque des enfants dans la pratique de I'AP, la 


\section{eJRIEPS 47 Juillet 2020}

théorie de l'autodétermination de Deci et Ryan peut être couplée au modèle socioécologique (Deci \& Ryan, 2008). En effet, les leviers d'intervention ont pour objectif de modifier les trois besoins psychologiques fondamentaux de l'enfant, c'est-à-dire le sentiment de compétence, d'autonomie et d'affiliation sociale. De ce fait, ces changements vont agir positivement sur la motivation de l'enfant à pratiquer une AP en favorisant le plaisir de la pratique et vont ainsi participer à la modification des comportements d'AP à long terme.

Les enseignants jouent un rôle primordial auprès des enfants et notamment sur ceux issus de milieux défavorisés. Situés sur le deuxième niveau du modèle socioécologique de Sallis et al. (2008), ils sont considérés comme l'un des facteurs sociaux ayant une influence cruciale sur les comportements des enfants (Eather, Morgan \& Lubans, 2013). Ainsi, leurs propres comportements liés à l'AP peuvent être déterminants sur les comportements actifs des enfants (Eather et al., 2013). Lors de la mise en place d'une intervention pour promouvoir l'AP et diminuer le TS en école primaire, ils peuvent être impliqués activement aux différentes actions (Kriemler \& al., 2011). Leur engagement est décisif, car il mène les enseignants à modifier leur pratique d'enseignement, à leur faire analyser de nouvelles situations et à les faire progresser (Uwamariya \& Mukamurera, 2005). Cependant les enseignants ne disposent pas toujours des compétences leur permettant de réaliser seuls des actions interventionnelles ou de choisir des contenus d'intervention pour promouvoir l'AP et diminuer le TS des enfants. En effet, les enseignants peuvent ne pas être suffisamment formés et peuvent manquer de soutien et de suivi lors de la mise en place des actions de promotion (Allisson \& al., 2016). Certains enseignants peuvent également éprouver un moindre intérêt pour ce domaine (Gilmore \& Donohoe, 2016). De ce fait, il est nécessaire pour l'équipe en charge d'un projet interventionnel d'apporter un soutien et un accompagnement professionnel aux enseignants, afin de faire entrer les enseignants dans une démarche de développement professionnel et de constituer un contexte favorable à la réussite de l'intervention (Beaudoin, Turcotte, Berrigan, Gignac \& Bernier, 2018 ; Turcotte, Gaudreau \& Otis, 2007). Suite à l'intervention, il est indispensable de comprendre quel a été leur rôle auprès des enfants, mais également de connaître leurs ressentis. Ainsi, il est possible de les interroger à propos des expériences interventionnelles qu'ils ont vécues au cours de l'intervention. Plusieurs indicateurs peuvent être ainsi mesurés par questionnaire : 1) Participation aux différentes actions, reflet de leur engagement ; 2) Perceptions à 


\section{eJRIEPS 47 Juillet 2020}

propos de l'efficacité des actions menées (évolution de l'AP et du TS de l'enfant, de son comportement en classe) ; 3) Modifications comportementales personnelles liées à l'AP (plaisir de pratiquer, évolution de la pratique au cours de l'intervention).

Moins de $10 \%$ des études interventionnelles en milieu scolaire ont été réalisées auprès d'enfants de quartiers défavorisés depuis la dernière décennie (Kriemler \& al., 2011). De plus, l'Organisation Mondiale de la Santé (OMS) en 2019 a pour objectif de diminuer la prévalence de l'inactivité physique chez les jeunes ainsi que diminuer les inégalités sociales. Prenant en considération tous ces points, une intervention a été dupliquée dans deux écoles primaires situées en Réseau d'Education Prioritaire ayant pour objectif de promouvoir l'AP et de diminuer le TS. Ces interventions ont combiné l'approche socio-écologique ainsi que la théorie de l'autodétermination (Deci \& Ryan, 2008 ; Sallis \& al., 2008). Les enseignants ont participé activement et ont été des facteurs fondamentaux dans la conception et dans la mise en place des actions interventionnelles. Ainsi, l'objectif de cette étude est d'analyser les expériences vécues des enseignants suite à l'intervention destinée à promouvoir l'AP et à diminuer le TS chez des enfants d'école primaire issus d'un quartier défavorisé.

\section{Méthodologie}

\subsection{Protocole expérimental de l'étude}

Deux écoles primaires de la ville de Tarbes (Hautes-Pyrénées, France) situées en Réseau d'Education Prioritaire ont été sollicitées et ont toutes les deux accepté de participer au projet d'intervention. Ce projet avait été discuté préalablement avec les équipes enseignantes des deux écoles qui s'étaient montrées volontaires. Ce projet d'intervention s'est effectué sur 3 années scolaires et un protocole expérimental a été parallèlement mis en place afin de mesurer les effets de l'intervention. Pendant la première année scolaire 2016/2017, aucune intervention n'a été effectuée. Sur cette même année, des mesures diagnostiques d'AP et de TS ont été effectuées en début d'année dans les deux écoles dans le cadre d'une première période de récolte de données notée $T 1$ («T $T$ » faisant référence à «temps de mesure »; T1, novembre 2016). Ces données ont été récoltées afin de connaître les niveaux d'AP et de TS initiaux des enfants, sans intervention préalable. En fin d'année scolaire, ces mesures ont été répétées afin d'observer un éventuel effet de saison (T2, juin 2017). Pendant la deuxième année scolaire 2017/2018, l'intervention destinée à promouvoir l'AP et 


\section{eJRIEPS 47 Juillet 2020}

diminuer le TS a été mise en place dans l'école 1. L'école 2 a été assignée comme école contrôle. De manière similaire à la première année du projet, des données d'AP et de TS des enfants ont été récoltées en début d'année scolaire, alors que les premiers ateliers de sensibilisation dans l'école 1 avaient commencé (T3, novembre 2017). Enfin, une quatrième période de récolte de données a été effectuée en postintervention (T4, juin 2018). Pendant la troisième année de l'étude 2018/2019, cette même intervention a été dupliquée dans l'école 2 ; une seule période de récolte de données a été effectuée dans les deux écoles en fin d'année scolaire (T5, juin 2019). Une équipe universitaire a participé à la mise en place du protocole expérimental et de l'intervention dans les deux écoles. Plus précisément, deux chercheurs, quatre étudiants et une doctorante diplômés dans le domaine de l'activité physique et de la santé ont participé aux différentes phases de récolte de données et sont également intervenus dans les écoles dans le cadre de l'intervention. Ils ont travaillé en collaboration avec l'ensemble des équipes enseignantes des deux écoles, l'Inspection Académique (IA) du département, et les politiques éducatives de la ville.

\subsection{Mesures réalisées}

À chaque période de récolte de données (T1, T2, T3, T4, T5), l'AP et le TS ont été évalués par accélérométrie. II s'agit d'une méthode de mesure objective valide permettant de recueillir pour chaque enfant des temps précis d'AP avec les intensités pratiquées, ainsi que le temps passé en position sédentaire. Les enfants devaient avoir obligatoirement une autorisation des parents pour porter l'accéléromètre pendant 7 jours sur chaque période. Des analyses du temps passé à pratiquer de l'AP et du TS sur une journée d'école classique et du week-end seront effectuées afin de déterminer les effets de chaque intervention sur les comportements actifs des enfants. À chaque période, d'autres données ont été récoltées par l'équipe universitaire en charge du projet. Un questionnaire destiné aux enseignants a notamment permis de recueillir les expériences vécues au cours de l'intervention, objet de cette étude.

\subsection{Implication des enseignants à l'intervention}

Tous les enfants du CP au CM2, âgés en moyenne de 6 à 12 ans, ont bénéficié des actions interventionnelles, ce qui correspond approximativement à 400 enfants par établissement. De plus, $55 \%$ des enfants de chaque école ont porté l'accéléromètre à chaque temps de mesure. Au total, six leviers d'intervention ont été sélectionnés et 


\section{eJRIEPS 47 Juillet 2020}

mis en place: 1. Ateliers de sensibilisation et pauses actives en classe pour les enfants ; 2. Réunions, newsletters et feedbacks pour les parents ; 3. Sessions de formation des enseignants ; 4. Aménagement environnemental et matériel (traçage au sol dans la cour de récréation) ; 5. Aménagement organisationnel (diminution du temps passé à la cantine, aires aménagées dans la cour) ; 6 . Collaboration avec les politiques locales et éducationnelles, notamment la mairie et l'IA des Hautes-Pyrénées. Ainsi, les facteurs des différents niveaux du modèle socio-écologique ont été pris en considération et de nombreuses actions interventionnelles ont été mises en place par l'équipe universitaire. L'ensemble de ces actions, répertoriées sur le modèle socioécologique, sont rapportées sur la Figure 1.

Les enseignants ont ainsi été impliqués dans la conception et la mise en place de l'intervention, et ont également participé activement à l'ensemble du protocole expérimental. Tout d'abord, l'un des leviers les plus importants était de proposer aux enseignants de chaque école quatre sessions de formation sur la promotion de l'AP et du TS, d'une durée de 3 heures chacune. Ces rencontres étaient formelles. Avec le soutien de I'IA des Hautes-Pyrénées, ces sessions étaient comptabilisées parmi les formations continues proposées chaque année aux enseignants. La doctorante et intervenante en charge du projet d'intervention a piloté et animé l'ensemble de ces sessions. Les thèmes suivants ont été abordés : 1. Connaissances sur les notions d'AP, de TS, leurs liens avec la santé et travail sur la pratique d'AP personnelle ; 2 . Techniques et outils pour promouvoir l'AP et diminuer le TS des enfants (pauses actives, ateliers de sensibilisation, séances d'AP, modification de l'organisation et de l'environnement de la classe, travail interdisciplinaire) avec attribution de conseils pratiques et personnalisés en fonction de l'âge des enfants ; 3. Facteurs sociaux des différents niveaux du modèle socio-écologique pouvant influencer la pratique d'AP des enfants ; 5. Informations à propos de l'intervention (dates des périodes de récolte de données et des ateliers, taux de participation des enfants, etc.). Ces sessions avaient principalement pour but de favoriser les échanges d'idées et de solutions entre enseignants, de susciter de l'intérêt pour l'AP et pour l'éducation à la santé et d'améliorer leur sentiment de compétence. Ces formations ont été réalisées au cours des années interventionnelles. Les enseignants ont pu bénéficier de documents de synthèse concernant les principales connaissances abordées, ainsi que des conseils d'ordre pratique. II a également été proposé aux enseignants de recevoir des 


\section{eJRIEPS 47 Juillet 2020}

documents supplémentaires par messagerie électronique. L'ensemble de ces documents a été rédigé par la doctorante-intervenante.

Les enseignants avaient la possibilité de réaliser toutes les actions interventionnelles qu'ils souhaitaient au cours de chaque année interventionnelle respective. Par exemple, ils pouvaient ajouter des jeux dans la cour de récréation, ajouter des cours d'Education Physique et Sportive (EPS) supplémentaires, réaliser un travail interdisciplinaire en classe en lien avec l'AP, proposer des ateliers de sensibilisation supplémentaires ainsi que des pauses actives, etc. Cependant, au début de chaque année interventionnelle, les intervenants ont réalisé les premiers ateliers de sensibilisation et les pauses actives en classe. Cet accompagnement avait pour objectif de mettre les enseignants en confiance en leur montrant le déroulement de ces ateliers. Ils ont ainsi pu observer l'organisation de ces activités, notamment les différentes techniques et exercices utilisés par les intervenants. Sur la suite de chaque année interventionnelle, les enseignants ont mis en place des ateliers de sensibilisation et des pauses actives sans la présence des intervenants. Les enseignants pouvaient cependant les solliciter afin d'obtenir diverses suggestions, conseils et informations, notamment au travers des sessions de formation. Un support à distance a également été proposé.

Un des buts recherchés par l'équipe universitaire (intervenants) en charge du projet était de rendre les enseignants autonomes dans la mise en place et la réalisation de ces actions tout au long de l'année interventionnelle, afin qu'ils puissent les reproduire au long terme. De ce fait, les intervenants n'étaient pas présents tous les jours dans les écoles : leur fréquence d'accompagnement dépendait des phases de l'intervention. $\mathrm{Au}$ début de l'intervention, ils étaient présents pour assurer l'ensemble des démonstrations, puis peu à peu, une plus grande autonomie a été laissée aux enseignants. II est également important de souligner que les équipes enseignantes des écoles ont été impliquées lors de chaque période de récolte de données, c'est-àdire pendant un à deux mois à chaque année scolaire parmi les 3 années du protocole expérimental. À chaque période de récolte de données, les enseignants de chaque classe ont ainsi adapté leurs leçons en classe pendant la distribution des accéléromètres. Ils ont également participé à la distribution et au recueil des autorisations parentales pour le port de l'accéléromètre ainsi que d'autres documents (lettres d'information pour les parents, documents avec résultats et feedbacks sur les niveaux d'AP, etc.). L'ensemble de ce projet ayant été collaboratif avec les politiques 


\section{eJRIEPS 47 Juillet 2020}

locales et régionales, les enseignants ont également participé à des réunions avec des représentants communaux et de l'IA.

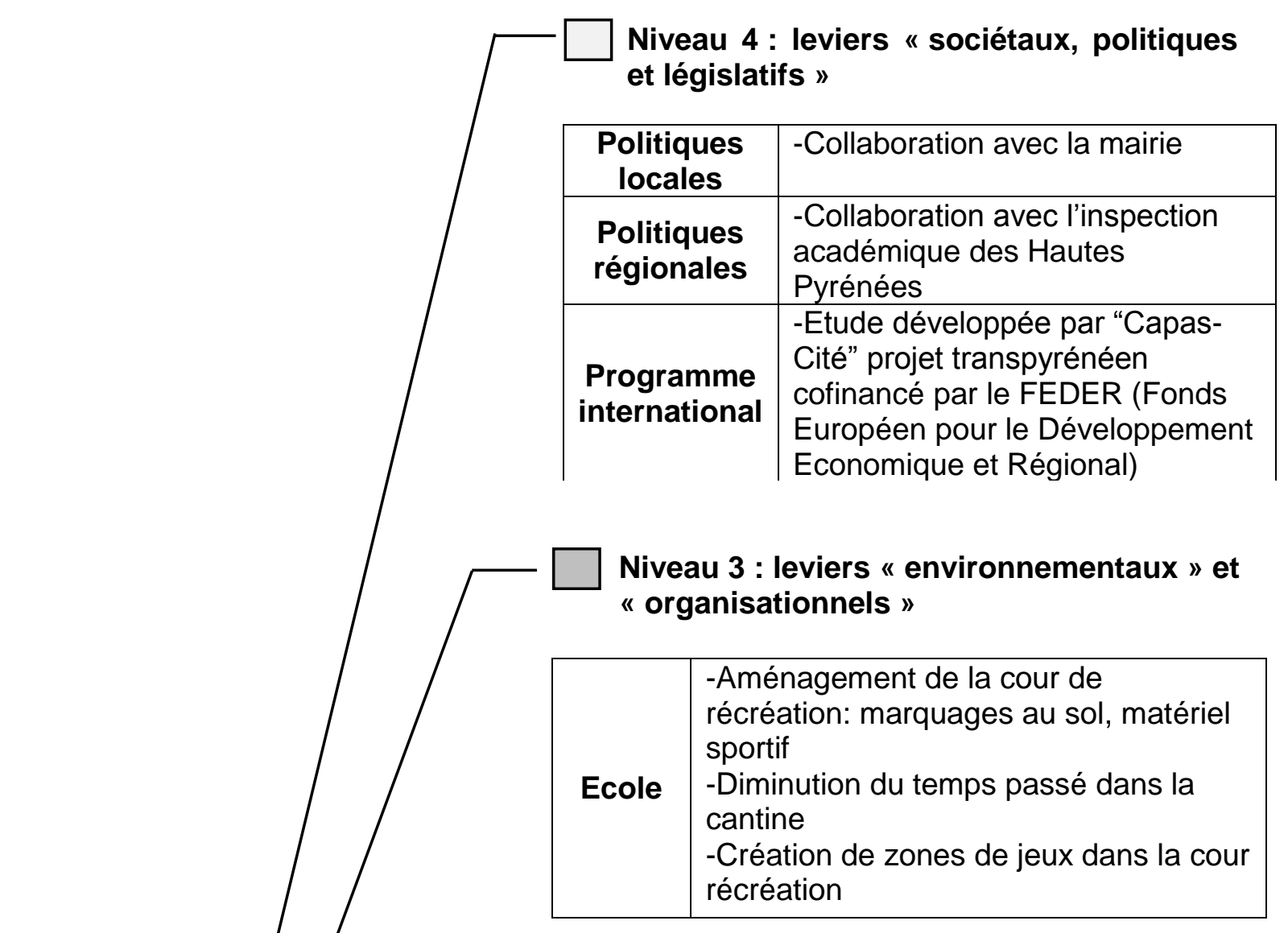

Niveau 2 : leviers interpersonnels

\begin{tabular}{|c|l|}
\hline Parents & $\begin{array}{l}\text {-Lettres d'information } \\
\text {-Feedbacks }\end{array}$ \\
\hline \multirow{5}{*}{ Enseignants } & $\begin{array}{l}\text {-Sessions de formation } \\
\text {-Participation active (ateliers de } \\
\text { sensibilisation, pauses } \\
\text { actives,...) } \\
\text {-Feedbacks }\end{array}$ \\
\hline
\end{tabular}

Niveau 1 : leviers intra-individuels

\begin{tabular}{|c|c|}
\hline Enfants & $\begin{array}{l}\text {-Ateliers de sensibilisation } \\
\text {-Séances d'activité physique et jeux } \\
\text { libres } \\
\text {-Pauses actives } \\
\text {-Feedbacks }\end{array}$ \\
\hline
\end{tabular}

Figure 1. Leviers d'intervention mobilisés sur les 4 niveaux du modèle socio-écologique dans les deux interventions scolaires. 


\section{eJRIEPS 47 Juillet 2020}

\subsection{Enseignants participants}

Un questionnaire a été réalisé avec l'interface «Google Forms » qui appartient à l'application «Google Drive». Les expérimentateurs peuvent ainsi rédiger des questions qui sont à choix simple ou multiples, ou pouvant demander une réponse ouverte courte ou longue. Le questionnaire a été élaboré par la doctoranteintervenante de l'équipe universitaire. Ce questionnaire était envoyé par messagerie électronique aux enseignants de chaque école l'année qui a suivi l'intervention. Ainsi, les enseignants de l'école 1 ont reçu le questionnaire au cours de l'année scolaire 2018/2019 alors que ceux de l'école 2 l'ont reçu plus tard en 2019/2020. Une période de latence de 5 mois a été appliquée entre la fin de l'intervention et l'envoi du questionnaire dans chaque école. Au maximum, trois rappels par messagerie électronique ont été effectués auprès des enseignants afin d'obtenir la totalité des réponses. Les réponses de chaque questionnaire étaient disponibles sur l'application «Google Drive » uniquement pour la doctorante-intervenante qui s'est chargés de leur diffusion auprès des enseignants.

La réalisation du questionnaire a pris entre 15 et 20 minutes à chaque enseignant. Leurs réponses étaient anonymes. La totalité des réponses a été traitée lorsque l'effectif complet des enseignants de chaque école ayant participé à l'intervention avait répondu au questionnaire. Le nombre de participants s'élève donc à 6 enseignants (5 femmes, 1 homme) pour l'école 1 et à 9 enseignants ( 8 femmes, 1 homme) dans l'école 2.

\subsection{Caractéristiques du questionnaire distribué aux enseignants}

Le questionnaire comportait quatre parties. Dans la première partie, les enseignants ont été interrogés à propos de leur participation sur la mise en place d'actions interventionnelles supplémentaires à celles réalisées par les intervenants. Ces questions concernaient la participation et la fréquence de réalisation des diverses actions interventionnelles : 1. Pauses actives pour diminuer le TS en classe ;2. Ateliers d'AP en classe et en récréation ; 3. Cours d'EPS. Si les enseignants n'ont pas effectué ces différentes actions, ils ont pu expliquer les raisons de ce choix. Deux questions supplémentaires ont été posées. L'une d'elle était destinée à savoir si les enseignants ont abordé le thème de l'AP et de la sédentarité avec les élèves. Une autre question avait pour objectif de savoir si les enseignants ont apporté d'éventuelles modifications dans l'organisation des leçons afin de proposer plus de mouvement aux enfants. Enfin, 


\section{eJRIEPS 47 Juillet 2020}

les enseignants ont également estimé leur investissement sur l'ensemble du projet, comprenant l'intervention et le protocole expérimental.

La deuxième partie du questionnaire s'est intéressée aux perceptions des enseignants concernant l'efficacité de l'intervention sur : 1. Les comportements d'AP des enfants ; 2. Les comportements sédentaires des enfants ; 3 . Le niveau de sensibilisation des enfants à ces problématiques. 4. D’autres caractéristiques (estime de soi, résultats scolaires, concentration des enfants en classe, etc.).

La troisième partie comportait des questions destinées à examiner les déterminants psychologiques des enseignants par rapport à l'intervention et à leur pratique personnelle d'AP. Plus précisément, les enseignants ont été interrogés sur le plaisir qu'ils ont éprouvé au cours de l'intervention. Ensuite, il a été demandé aux enseignants s'ils pensent avoir eu les compétences nécessaires pour réaliser les actions interventionnelles. Enfin, une dernière question sur cette troisième partie avait pour objectif de savoir si les enseignants se sont perçus comme étant un soutien essentiel pour les enfants leur permettant d'influencer leur pratique d'AP.

Dans une dernière partie, les enseignants ont pu faire part de l'évolution concernant leur propre niveau d'AP : 1. Plaisir à pratiquer ; 2. Evolution du temps de pratique d'AP ; 3. Evolution de la vision de l'AP et du TS. Enfin, les enseignants ont pu compléter deux questions ouvertes les invitant à mentionner des aspects de l'intervention qui auraient pu être améliorés ainsi que des nouveaux éléments interventionnels qui pourraient être ajoutés dans les futures interventions en école primaire.

\subsection{Exploitation des données}

Les réponses des enseignants à chaque question ont été reportées sur un fichier Excel. Pour les données quantitatives, des statistiques descriptives simples (moyenne, écart-type) ont été effectuées. Ces analyses ont été élaborées à l'aide des outils statistiques proposés par Google Forms. Ainsi, les statistiques descriptives ont permis d'explorer les réponses des données quantitatives des quatre parties du questionnaire. Elles ont été réalisées rigoureusement par la doctorante-intervenante. Le recueil des données sur le fichier Excel était la seule condition préalable à la réalisation des statistiques descriptives. Les données qualitatives, recueillies par les questions demandant une réponse ouverte courte ou longue, n'ont pas fait l'objet de méthodes statistiques et ont été reportées avec exactitude sur le fichier de données. Elles n'ont 


\section{eJRIEPS 47 Juillet 2020}

pas été soumises à une analyse complémentaire et servaient à appuyer les données quantitatives obtenues par accélérométrie. Les réponses des enseignants de deux écoles ont été analysées séparément afin de pouvoir disposer de retours d'expériences spécifiques à chaque contexte scolaire.

\section{Résultats : réponses des enseignants au questionnaire}

\subsection{Participation des enseignants aux actions interventionnelles}

Les résultats sont rapportés dans le tableau 1. Dans l'école 1, la moitié des enseignants (50\%) a mis en place des pauses actives en classe pour réduire le TS et les a réalisées quotidiennement. Dans l'école 2, les pauses actives ont été effectuées par une majorité d'enseignants (77,8\%). Parmi ces $77,8 \%$, seulement $28,6 \%$ d'entre eux les ont effectuées quotidiennement, contre $42,9 \%$ de manière hebdomadaire. De plus, parmi les $66,7 \%$ d'enseignants ayant réalisé des ateliers d'AP supplémentaire dans l'école 1, $50 \%$ les ont mis en place tous les jours et $25 \%$ de manière hebdomadaire. Dans l'école 2, parmi les 55,6\% des enseignants ayant effectué des ateliers d'AP, $40 \%$ les ont mis en place quotidiennement contre $60 \%$ de manière hebdomadaire. Des enseignants des deux écoles ont évoqué les raisons qui ont constitué un blocage à la réalisation des pauses actives pour réduire le TS. Parmi celles-ci, ils citent un « problème de place », un « problème de retour au calme » ainsi qu'« un manque de temps ». Parmi les enseignants qui n'ont pas effectué d'ateliers d'AP avec les enfants, le manque de temps est l'unique raison évoquée.

Dans l'école 1, aucun enseignant n'a mis en place des séances supplémentaires d'EPS avec les élèves au cours de l'année interventionnelle, alors que cela a été le cas pour 44,4\% des enseignants de l'école 2. Les enseignants de l'école 2 n'ayant pas pu réaliser des séances supplémentaires d'EPS citent également un manque de temps prévu pour l'EPS au cours de la semaine scolaire.

Au cours de l'intervention, $60 \%$ des enseignants de l'école 1 et 100\% des enseignants de l'école 2 ont abordé le thème de l'AP et de la sédentarité avec les élèves en classe. De plus, $66,7 \%$ des enseignants de l'école 1 et $88,9 \%$ des enseignants de l'école 2 ont modifié, ponctuellement ou quotidiennement, la manière d'organiser la classe et les enseignements afin de favoriser le mouvement chez les enfants.

Sur une échelle de 1 à 10 , les enseignants devaient également juger leur investissement à l'intervention avec une échelle allant de 1 (faible investissement) à 


\section{eJRIEPS 47 Juillet 2020}

10 (très grand investissement). Au total, 66,7\% des enseignants de l'école 1 ont estimé leur investissement dans l'intervention à 7 sur 10 sur cette échelle. De plus, 16,7\% des enseignants ont jugé leur investissement à 8 sur 10, et également 16,7\% l'ont estimé à 10 sur 10. Dans l'école 2, 66,8\% des enseignants ont jugé que leur investissement se situait au minimum à 8 sur 10 dont un qui estime se situer à 10 sur 10 (11,1\%).

Tableau I. Taux de participation des enseignants aux actions interventionnelles

\begin{tabular}{|c|c|c|c|c|c|c|}
\hline \multirow[b]{2}{*}{ Questions } & \multicolumn{3}{|c|}{ Ecole 1} & \multicolumn{3}{|c|}{ Ecole 2} \\
\hline & «Oui » & $\begin{array}{l}\mathrm{Si} \text { «Oui », à } \\
\text { quelle } \\
\text { fréquence? }\end{array}$ & «Non » & «Oui » & $\begin{array}{l}\mathrm{Si} \text { ¿ Oui », à } \\
\text { quelle } \\
\text { fréquence? }\end{array}$ & «Non » \\
\hline $\begin{array}{l}\text { Avez-vous réalisé } \\
\text { des breaks } \\
\text { (pauses actives) } \\
\text { pour diminuer le } \\
\text { temps sédentaire } \\
\text { en classe? }\end{array}$ & $50 \%$ & $\begin{array}{l}100 \% \text { des } \\
\text { répondants } \\
\text { " oui » les } \\
\text { ont réalisés } \\
\text { quotidienne } \\
\text { ment }\end{array}$ & $50 \%$ & $77,8 \%$ & $\begin{array}{l}28,6 \% \text { des } \\
\text { répondants } \\
\text { « oui » les } \\
\text { ont réalisés } \\
\text { quotidiennem } \\
\text { ent ; } 42,9 \% \\
\text { de manière } \\
\text { hebdomadair } \\
\text { e } ; 28,6 \% \text { en } \\
\text { fonction du } \\
\text { besoin des } \\
\text { élèves }\end{array}$ & $22,8 \%$ \\
\hline $\begin{array}{l}\text { Avez-vous réalisé } \\
\text { des ateliers afin } \\
\text { de favoriser } \\
\text { l'activité physique } \\
\text { des enfants (en } \\
\text { classe, en } \\
\text { récréation, sans } \\
\text { compter des } \\
\text { séances d'EPS } \\
\text { supplémentaires) } \\
\text { ? }\end{array}$ & $66,7 \%$ & $\begin{array}{l}50 \% \text { des } \\
\text { répondants } \\
\text { « Oui » les } \\
\text { ont réalisés } \\
\text { quotidienne } \\
\text { ment ; } 50 \% \\
\text { de manière } \\
\text { hebdomadai } \\
\text { re ; } 28,6 \% \\
\text { de manière } \\
\text { mensuelle }\end{array}$ & $33,3 \%$ & $55,6 \%$ & $\begin{array}{l}40 \% \text { des } \\
\text { répondants } \\
\text { « oui » les } \\
\text { ont réalisés } \\
\text { quotidiennem } \\
\text { ent ; } 60 \% \text { de } \\
\text { manière } \\
\text { hebdomadair } \\
\text { e }\end{array}$ & $44,4 \%$ \\
\hline $\begin{array}{l}\text { Avez-vous réalisé } \\
\text { des séances } \\
\text { supplémentaires } \\
\text { d'EPS? }\end{array}$ & $0 \%$ & $\begin{array}{l}\text { Pas de } \\
\text { réponses }\end{array}$ & $100 \%$ & $44,4 \%$ & $\begin{array}{l}75 \% \text { des } \\
\text { répondants } \\
\text { « oui » les } \\
\text { ont réalisés } \\
\text { de manière } \\
\text { hebdomadair } \\
\text { e ; } 25 \% \text { à } \\
\text { certaines } \\
\text { périodes. }\end{array}$ & $55,6 \%$ \\
\hline
\end{tabular}




\section{eJRIEPS 47 Juillet 2020}

\subsection{Perceptions de l'efficacité de l'intervention}

Les résultats complets sont présentés dans le tableau 2. Au total, $75 \%$ des enseignants de l'école 1 et $62,5 \%$ des enseignants de l'école 2 pensent que l'intervention a permis à plus de la moitié des enfants de modifier leurs comportements liés à la pratique d'AP. De plus, $60 \%$ des enseignants de l'école 1 et $55,5 \%$ des enseignants de l'école 2 pensent que l'intervention a été bénéfique également sur les comportements sédentaires pour plus de la moitié des enfants. Enfin, $80 \%$ des enseignants de l'école 1 ainsi que $77,7 \%$ des enseignants de l'école 2 pensent que l'intervention a permis également à plus de la moitié des enfants d'être plus sensibilisés aux effets de l'AP et de la sédentarité sur la santé.

Les enseignants ont évoqué d'autres effets bénéfiques de l'intervention. En effet, dans l'école $1,50 \%$ des enseignants ont rapporté une diminution des tensions entre les enfants, $25 \%$ des enseignants ont estimé que l'intervention a permis une diminution du stress des enfants, et $25 \%$ ont également indiqué une meilleure concentration des enfants sur les tâches scolaires. Dans l'école 2, 50\% des enseignants mentionnent une meilleure concentration sur des tâches scolaires ainsi que d'autres effets bénéfiques tels qu'une amélioration de l'estime de soi des enfants, des capacités motrices et une diminution du stress.

Enfin, respectivement, $80 \%$ et $88,9 \%$ des enseignants de l'école 1 et de l'école 2 pensent que cette intervention va modifier leurs pratiques d'enseignement en classe et en EPS au long terme. 


\section{eJRIEPS 47 Juillet 2020}

Tableau II. Effets de l'intervention sur le comportement des enfants d'après les enseignants

\begin{tabular}{|c|c|c|c|c|c|c|}
\hline & \multicolumn{2}{|c|}{$\begin{array}{c}\text { Question 1: } \\
\text { Pensez-vous que } \\
\text { l'intervention a } \\
\text { permis aux enfants } \\
\text { de modifier leurs } \\
\text { comportements en } \\
\text { lien avec l'activité } \\
\text { physique? }\end{array}$} & \multicolumn{2}{|c|}{$\begin{array}{c}\text { Question 2 : } \\
\text { Pensez-vous que } \\
\text { l'intervention a } \\
\text { permis aux enfants } \\
\text { de modifier leurs } \\
\text { comportements en } \\
\text { lien avec la } \\
\text { sédentarité ? }\end{array}$} & \multicolumn{2}{|c|}{$\begin{array}{c}\text { Question 3 : } \\
\text { Pensez-vous que } \\
\text { l'intervention a } \\
\text { permis aux enfants } \\
\text { d'être plus } \\
\text { sensibilisés par } \\
\text { rapport aux effets de } \\
\text { l'activité physique et } \\
\text { de la sédentarité sur } \\
\text { la santé ? }\end{array}$} \\
\hline Réponses & Ecole 1 & Ecole 2 & Ecole 1 & Ecole 2 & Ecole 1 & Ecole 2 \\
\hline $\begin{array}{l}\text { «Oui, pour la } \\
\text { totalité des } \\
\text { enfants de } \\
\text { l'école » }\end{array}$ & $0 \%$ & $0 \%$ & $0 \%$ & $0 \%$ & $0 \%$ & $22,2 \%$ \\
\hline $\begin{array}{l}\text { «Oui, pour les } \\
\text { trois quarts } \\
\text { des enfants } \\
\text { de l'école» }\end{array}$ & $25 \%$ & $12,5 \%$ & $20 \%$ & $22,2 \%$ & $20 \%$ & $33,3 \%$ \\
\hline $\begin{array}{l}\text { «Oui, pour la } \\
\text { moitié des } \\
\text { enfants de } \\
\text { l'école » }\end{array}$ & $50 \%$ & $50 \%$ & $40 \%$ & $33,3 \%$ & $60 \%$ & $22,2 \%$ \\
\hline $\begin{array}{l}\text { «Oui, pour } \\
\text { moins de la } \\
\text { moitié des } \\
\text { enfants de } \\
\text { l'école » }\end{array}$ & $25 \%$ & $37,5 \%$ & $40 \%$ & $33,3 \%$ & $20 \%$ & $22,2 \%$ \\
\hline $\begin{array}{l}\text { «on, pas du } \\
\text { tout » }\end{array}$ & $0 \%$ & $0 \%$ & $20 \%$ & $11,1 \%$ & $20 \%$ & $0 \%$ \\
\hline
\end{tabular}

\subsection{Déterminants psychologiques des enseignants}

L'ensemble des enseignants des deux écoles (100\%) déclare avoir pris du plaisir à participer à l'intervention. Les enseignants de l'école 2 évoquent le fait que l'intervention a pu « retravailler certains dispositifs ». Un autre enseignant évoque le fait qu'il « faut le temps d'avoir un groupe classe prêt à fonctionner ». Respectivement, $83,3 \%$ et $87,5 \%$ des enseignants de l'école 1 et de l'école 2 pensent avoir eu les compétences nécessaires pour participer à ce projet d'intervention. Enfin, dans l'école 1 et dans l'école 2,50\% des enseignants ont indiqué le fait qu'ils ne pensent pas avoir été un soutien important pour les enfants. D’autre part, un enseignant mentionne avoir 


\section{eJRIEPS 47 Juillet 2020}

été « un soutien au moins dans la prise de conscience de la nécessité d'intégrer l'AP dans le mode de vie traditionnel ». Un enseignant évoque avoir été « un soutien » pour impulser des choses mais il explique qu'il faut un relai à l'extérieur comme les parents et les personnels de garderies extrascolaires.

\subsection{Evolution du niveau d'AP personnel}

L'évolution des comportements actifs des enseignants suite aux interventions est présentée dans le tableau 3 . Au total, $100 \%$ des enseignants de l'école 1 et $88,9 \%$ des enseignants de l'école 2 prennent du plaisir à pratiquer de l'AP. De plus, 33,3\% des enseignants de l'école 1 et $44,4 \%$ des enseignants de l'école 2 ont augmenté leur temps de pratique d'AP au cours de l'intervention. Parmi les $33,3 \%$ des enseignants de l'école 1,50\% des enseignants l'ont augmenté de moins de 30 minutes et $50 \%$ l'ont augmenté entre 30 minutes et 2 heures par semaine. Dans l'école 2, 44,4\% des enseignants ont augmenté leur temps de pratique de 30 minutes à 2 heures supplémentaires hebdomadaire. Au total, $80 \%$ des enseignants de l'école 1 et $66,7 \%$ des enseignants de l'école 2 avaient la même vision de l'AP et du sport en pré intervention et en post intervention. Les enseignants ont ensuite décrit leur vision de l'AP et du sport. Dans l'école 1, ils y associent la santé, le bien-être, la socialisation, la détente, le plaisir, le fait de "se sentir mieux dans son corps et dans sa tête ". Dans l'école 2, deux enseignants mentionnent le caractère indispensable de l'AP mais également du besoin de disposer de temps pour en effectuer. 
Tableau III. Evolution des comportements actifs des enseignants suite à l'intervention

\begin{tabular}{|l|l|l|l|l|}
\cline { 2 - 5 } \multicolumn{1}{c|}{} & \multicolumn{2}{c|}{ Ecole 1 } & \multicolumn{2}{c|}{ Ecole 2 } \\
\hline \multicolumn{1}{c|}{ Questions } & $\begin{array}{l}\text { Réponse : } \\
\text { «Oui » }\end{array}$ & $\begin{array}{l}\text { Réponse : } \\
\text { « Non » }\end{array}$ & $\begin{array}{l}\text { Réponse : } \\
\text { «Oui » }\end{array}$ & $\begin{array}{l}\text { Réponse : } \\
\text { « Non » }\end{array}$ \\
\hline $\begin{array}{l}\text { Actuellement, } \\
\text { prenez-vous du } \\
\text { plaisir à pratiquer } \\
\text { de l'activité } \\
\text { physique? }\end{array}$ & $100 \%$ & $0 \%$ & $88,9 \%$ & $22,2 \%$ \\
\hline $\begin{array}{l}\text { Au cours de } \\
\text { l'intervention, } \\
\text { avez-vous } \\
\text { augmenté votre } \\
\text { temps de pratique } \\
\text { d'activité } \\
\text { physique? }\end{array}$ & $33,3 \%$ & $66,7 \%$ & $44,4 \%$ & $55,6 \%$ \\
\hline $\begin{array}{l}\text { Avant } \\
\text { l'intervention, } \\
\text { aviez-vous une } \\
\text { autre vision de } \\
\text { l'activité physique } \\
\text { du sport, de la } \\
\text { sédentarité ? }\end{array}$ & $20 \%$ & $80 \%$ & $33,3 \%$ & $66,7 \%$ \\
\hline
\end{tabular}

\subsection{Remarques des enseignants}

Les enseignants ont souligné l'importance d'élargir l'intervention aux équipes périscolaires de l'école. Un enseignant évoque la difficulté de «transposer l'intervention à la maison sans une sensibilisation des familles » et le « besoin d'avoir des feedbacks supplémentaires sur la pratique des enfants ». De plus, un enseignant a souligné que "la réalisation des pauses actives pour diminuer le TS a été très motivante ».

\section{Discussion}

L'objectif de cet article était d'analyser les expériences vécues par les enseignants de deux écoles suite à une intervention destinée à promouvoir l'AP et diminuer le TS pour les enfants. En effet, celle-ci a été mise en place successivement dans deux écoles primaires situées en Réseau d'Education Prioritaire dans la ville de Tarbes. Jusqu'à présent, peu d'études se sont focalisées sur les expériences vécues par les enseignants lors d'une intervention destinée à promouvoir l'AP et diminuer le TS. Or, ces données permettent de mieux comprendre leur rôle auprès des enfants au cours 


\section{eJRIEPS 47 Juillet 2020}

de l'intervention. Plusieurs indicateurs ont été retenus afin de rassembler les expériences vécues: taux de participation et engagement des enseignants, leur perception liée à l'efficacité de l'intervention, compétences perçues, pratique d'AP individuelle. Certains des indicateurs mesurés, tels que l'engagement ainsi que la pratique personnelle d'AP de l'enseignant peuvent être de potentielles variables pouvant influencer les comportements actifs des enfants (Eather \& al., 2013 ; Uwamariya \& Mukamurera, 2005). II a été remarqué que les enseignants ont adhéré au projet d'intervention en proposant en toute autonomie des pauses actives pour diminuer le TS ainsi que des ateliers d'AP supplémentaires. Une majorité d'enseignants a ainsi modifié ses pratiques d'enseignement au cours de l'intervention pour favoriser le mouvement chez les enfants. Cependant, le manque de temps semble être la barrière la plus importante pour la réalisation des ateliers d'EPS supplémentaires et constitue une des limites fréquemment rencontrée dans les études interventionnelles (Benes, Fin, Sullivan \& Yan, 2016). II est possible que la charge importante des programmes scolaires ne laisse pas la possibilité aux enseignants d'effectuer des séances d'EPS qui demandent entre 1 heure à 2 heures par séance. Les ateliers d'AP, d'une durée moindre, semblent avoir été épargnés par ce manque de temps. L'étude interventionnelle de Mc Mullen et al. (2014) s'était focalisée sur les perceptions des enseignants au cours d'une intervention et il en est ressorti que les enseignants ont préféré réaliser des pauses actives qui étaient plus faciles à mettre en place et qui étaient plus rapides et amusantes pour les enfants. Ce résultat rejoint les constats de notre étude.

De plus, nous pouvons constater que les enseignants de l'école 2 ont été plus nombreux à effectuer des pauses actives et des séances d'EPS que les enseignants de l'école 1. De nombreuses variables peuvent expliquer ces résultats, qui s'avèrent être spécifiques au contexte de chaque école. II semble, par exemple, que les enseignants de l'école 2, qui étaient en présence d'enfants plus défavorisés que ceux de l'école 1, aient recherché davantage de solutions pour modifier positivement le climat de la classe et favoriser des apprentissages liés à l'éducation à la santé. Ainsi, ils ont possiblement mis à profit les outils théoriques et pratiques qui leur ont été proposés par l'équipe universitaire en charge du projet face aux besoins importants de ces élèves en matière d'apprentissage. D'autre part, ce questionnaire a indiqué que la majorité des enseignants a observé et rapporté des effets bénéfiques sur les comportements d'AP et de TS de plus de la moitié des enfants. II a également indiqué 


\section{eJRIEPS 47 Juillet 2020}

que les enfants se concentraient mieux en classe lors de l'année interventionnelle. Or, ce constat a également été réalisé dans l'étude de Turcotte et al. (2012) qui avait étudié les perceptions d'enseignantes lors de la mise en place d'une période d'activité physique quotidienne dans une école canadienne. Les enseignants semblent convaincus des effets de l'intervention sur les comportements des enfants. Or, la littérature scientifique indique que les enseignants modifient leurs pratiques uniquement s'ils sont convaincus des effets du projet qu'ils portent sur les apprentissages des enfants (Stylianou \& al., 2016 ; VanTassel-Baska \& al., 2008). Ainsi, au cours de l'intervention, les enseignants ayant observé des modifications comportementales positives des enfants ont sans doute continué à modifier leur pratique d'enseignement jusqu'à la fin de l'intervention.

II est important de mentionner que des sessions de formation pour les enseignants avaient également été mises en place afin de les accompagner au cours de l'intervention. Or, ce suivi est essentiel et constitue un des piliers sur lesquels les enseignants peuvent développer de nouvelles compétences en matière d'éducation à la santé (Beaudoin \& al., 2018 ; Benes \& al., 2016). Les travaux menés par l'équipe de Turcotte montrent que les enseignants doivent être au cœur de toute démarche interventionnelle (Turcotte \& al., 2007). De ce fait, les sessions de formation continue effectuées pour les enseignants ainsi que le suivi régulier organisé pendant les phases de mesure ont participé au développement professionnel des enseignements, qui est une des conditions de la réussite de l'intervention (Beaudoin \& al., 2018). Les sessions de formation se déroulaient en groupes, entre collègues, et poussaient les enseignants à échanger entre eux et à trouver des stratégies efficaces et spécifiques à chaque école. Or, ces ateliers en groupe permettent à chaque enseignant de repenser leurs pratiques d'enseignement et de créer une démarche de changements collectifs (Uwamariya \& Mukamurera, 2005). De plus, les enseignants étaient impliqués sur la totalité du protocole expérimental d'une durée de 3 ans. Ils ont ainsi participé activement aux périodes de récolte de données. Cette émulation de tous les enseignants sur la durée totale de ce projet a sans doute constitué un facteur important contribuant à la réussite de ce projet d'intervention. L'accompagnement professionnel et la cohésion de groupe a ainsi pu favoriser une augmentation du sentiment de compétence des enseignants envers les actions interventionnelles.

Cependant, peu d'enseignants se sont considérés comme des soutiens importants pour promouvoir les comportements actifs chez les enfants. Or, le soutien des 


\section{eJRIEPS 47 Juillet 2020}

enseignants apparaît comme le plus important facteur médiateur de l'intervention sur I'AP des enfants comparativement au soutien perçu des parents ou des amis (Eather \& al., 2013). Cette perception liée au faible soutien peut avoir été une source de démotivation chez les enseignants. II est possible que cette perception ait limité leurs actions interventionnelles. Lors des sessions de formation, leur rôle de soutien avait été expliqué et valorisé. Néanmoins, il semble qu'ils avaient déjà attribué un rôle important à l'éducation parentale comme facteur explicatif déterminant sur les comportements actifs des enfants. II aurait été judicieux d'insister plus longuement sur l'importance de leur rôle de soutien et de l'impact qu'ils peuvent avoir auprès des enfants. Cela aurait permis de renforcer leur confiance en soi au cours de l'intervention. II est possible que les enseignants les plus impliqués personnellement dans la pratique d'AP et ayant également pris du plaisir à participer à l'intervention aient favorisé les comportements actifs chez les enfants.

Les échanges effectués avec les équipes enseignantes avant la totalité du projet (protocole expérimental et intervention) ont permis de constater qu'elles étaient motivées dès le départ. Elles suscitaient déjà un intérêt pour la promotion de l'AP. Cependant, il aurait été judicieux de recueillir plus spécifiquement leurs besoins et leurs attentes pour réaliser un suivi plus individualisé. De plus, il aurait été intéressant de recueillir des informations à propos de leur vision de l'AP et de leur sentiment de compétence liés à la conduite de séances d'AP avant la conduite de ce projet. Cette évaluation préalable aurait permis de mieux connaître l'évolution des comportements des enseignants vis-à-vis de leurs propres expériences d'AP et de leurs pratiques d'enseignement liées à la promotion de l'AP.

Bien que les pratiques d'enseignements aient été modifiées au cours de l'intervention, il est pertinent de se demander si les enseignants vont poursuivre les actions qu'ils ont menées ou s'ils vont revenir à leurs anciennes habitudes une fois l'intervention terminée. Au cours de cette étude, $80 \%$ des enseignants de l'école 1 et $88,9 \%$ des enseignants de l'école 2 pensent que l'intervention va modifier leurs pratiques d'enseignement en classe et en EPS au long terme. Le suivi actif des enseignants et à plusieurs reprises au cours de l'intervention a pu participer à la pérennisation des nouvelles habitudes des enseignants.

II serait pertinent, dans la continuité de notre étude, de proposer un autre questionnaire aux enseignants 2 ans à 5 ans suivant la fin de l'intervention. Cela permettrait de connaître les effets de l'intervention sur l'évolution de leurs techniques d'enseignement 


\section{eJRIEPS 47 Juillet 2020}

au long terme liées à la promotion des comportements actifs. Ces informations seront essentielles afin de savoir si les enseignants, qui disposent de 3 heures dans leur service pour l'EPS, peuvent réaliser de multiples actions auprès des enfants et de leurs familles ou si des intervenants extérieurs semblent nécessaires pour les assurer. Des actions sortant du cadre scolaire sont également requises, notamment auprès des équipes périscolaires qui font le lien entre l'AP scolaire et l'AP extrascolaire. En effet, les habitudes de vie doivent également être modifiées en dehors des heures scolaires, représentant un grand défi de la promotion de l'AP auprès des enfants.

\section{Conclusion}

Dans cette étude, les expériences vécues par les enseignants au cours d'une intervention multi-levier visant à promouvoir l'AP et diminuer le TS en école primaire d'un quartier défavorisé ont été explorées. De manière générale, ces données ont permis de connaître le niveau d'investissement des enseignants à l'intervention, leur perception concernant l'efficacité de l'intervention ainsi que leur rapport personnel à la pratique de l'AP. Ces données ont été recueillies en complément des mesures d'AP et de TS effectuées par accélérométrie et qui étaient destinées à évaluer les effets de l'intervention sur les comportements actifs des enfants. Peu d'études interventionnelles se focalisent sur les perceptions des enseignants au cours d'une intervention. En effet, elles s'intéressent uniquement aux résultats d'AP obtenus par accéléromètre sans tenir compte des facteurs humains pouvant influencer ces données objectives. Or, ces données permettent de contribuer à l'avancement des connaissances actuelles relatives aux interventions visant à promouvoir les comportements de santé des enfants, notamment celles qui se déroulent en école primaire et qui impliquent une participation active des enseignants. Ces données ont également permis de pointer les dysfonctionnements liés à l'implémentation des différentes actions. Elle a ainsi ouvert la voie à des pistes d'amélioration pour les futures études interventionnelles multi-leviers dont le rôle des enseignants s'avère crucial. Bien que les expériences aient été recueillies dans un contexte scolaire bien précis, cet article constitue une source d'information pour les enseignants et les politiques liées à l'éducation et à la santé. En finalité, ce partage d'expérience peut permettre de progresser dans le domaine de la promotion de l'AP auprès des enfants d'école primaire. 


\section{eJRIEPS 47 Juillet 2020}

\section{Bibliographie}

Allison, K. R., Vu-Nguyen, K., Ng, B., Schoueri-Mychasiw, N., Dwyer, J. J., Manson, H., Hobin, E., Manske, S., \& Robertson, J. (2016). Evaluation of daily physical activity (DPA) policy implementation in Ontario: Survey of elementary school administrators and teachers. BMC Public Health, 16, 746-761.

Beaudoin, S., Turcotte, S., Berrigan, F., Gignac, C., \& Bernier, P. M. (2018). Dispositif d'accompagnement professionnel pour optimiser la mise en œuvre d'une période d'activité physique quotidienne en milieu scolaire. Staps, 2, 63-81.

Benes, S., Finn, K. E., Sullivan, E. C., \& Yan, Z. (2016). Teachers' perceptions of using movement in the classroom. Physical Educator, 73(1), 110-135.

Boutet, M. \& Villemin, R. (2014). L'accompagnement : un élément clé pour l'apprentissage en stage et pour le développement professionnel continu des enseignants. Phronesis, 3(1-2), 81-89.

Cheval, B., Radel, R., Neva, J. L., Boyd, L. A., Swinnen, S.P., Sander, D., \& Boisgontier, M.P. (2018). Behavioral and neural evidence of the rewarding value of exercise behaviors: a systematic review. Sports Medicine, 48(6), 1389-1404.

Cheval, B., Sieber, S., Guessous, I., Orsholits, D., Courvoisier, D. C., Kliegel, M., ... \& Boisgontier, M. (2018). Effect of early-and adult-life socioeconomic circumstances on physical inactivity. Medicine and science in sports and exercise, 50(3), 476-485.

Cregan-Reid, V. (2018). Primate Change: How the world we made is remaking us. Hachette UK.

Deaudelin, C., Brodeur, M., \& Bru, M. (2005). Conclusion: un portrait caractéristique de la recherche sur le développement professionnel des enseignants et sur la formation à l'enseignement. Revue des sciences de l'éducation, 31(1), 177-185.

Deci, E. L., Ryan, R. M. (2008). Self-determination theory: A macrotheory of human motivation, development, and health. Canadian psychology/Psychologie canadienne, 49(3), 182-185.

Eather, N., Morgan, P. J., \& Lubans, D. R. (2013). Social support from teachers mediates physical activity behavior change in children participating in the Fit4-Fun intervention. International Journal of Behavioral Nutrition and Physical Activity, 10(1), 1-15. 


\section{eJRIEPS 47 Juillet 2020}

Gilmore, T. \& Donohoe, H. (2016). Elementary school generalist teachers' perceived competence to deliver Ontario's daily physical activity program. Society and Leisure, 39, 135-144.

Jones R. A., Hinkley, T, Okely, A. D, \& Salmon J. (2013). Tracking physical activity and sedentary behavior in childhood: a systematic review. American Journal of Preventive Medicine, 44(6), 651-658.

King, K. M., \& Ling, J. (2015). Results of a 3-year, nutrition and physical activity intervention for children in rural, low-socioeconomic status elementary schools. Health education research, 30(4), 647-659.

Kriemler, S., Meyer, U., Martin, E., van Sluijs, E. M., Andersen, L. B., \& Martin, B. W. (2011). Effect of school-based interventions on physical activity and fitness in children and adolescents: a review of reviews and systematic update. British journal of sports medicine, 45(11), 923-930.

Martin, R., \& Murtagh, E. (2017). Active classrooms: a cluster randomized controlled trial evaluating the effects of a movement integration intervention on the physical activity levels of primary school children. Journal of Physical Activity and Health, 14(4), 290-300.

McMullen, J., Kulinna, P., \& Cothran, D. (2014). Physical activity opportunities during the school day: classroom teachers' perceptions of using activity breaks in the classroom. Journal of Teaching in Physical Education, 33(4), 511-527.

Perkins, D. D., Meeks, J.W., \& Taylor, R. B. (1992). The physical environment of street blocks and resident perceptions of crime and disorder: Implications for theory and measurement. Journal of Environmental Psychology, 12(1), 21-34.

Rapport de Santé Publique France. (2015). Étude de santé sur l'activité physique (Esteban).https://www.santepubliquefrance.fr/determinants-desante/nutrition-et-activite-physique/documents/rapport-synthese/etude-desante-sur-I-environnement-la-biosurveillance-I-activite-physique-et-lanutrition-esteban-2014-2016.-volet-nutrition.-chapitre-activit Consulté le 02 janvier 2020.

Sallis, J. F., Owen, N., \& Fisher, E. B. (2008). Ecological models of health behavior. Dans K. Glanz, B.K. Rimer \& K. Viswanath (Eds.), Health behavior and health education: theory, research and practice (p. 465-485), 4ème éd. San Francisco, CA: Jossey-Bass. 


\section{eJRIEPS 47 Juillet 2020}

Sanders-Phillips, K. (2000). The Impact of Exposure to Violence. Promoting human wellness: New frontiers for research, practice, and policy, 294-310.

Stylianou, M., Kulinna, P. H., \& Naiman, T. (2016). '... because there's nobody who can just sit that long' Teacher perceptions of classroom-based physical activity and related management issues. European Physical Education Review, 22(3), 390-408.

Turcotte, S., Gaudreau, L., \& Otis, J. (2007). Démarche de modélisation de l'intervention en éducation à la santé incluse en éducation physique. Staps, 3, 63-78.

Uwamariya, A. \& Mukamurera, J. (2005). Le concept de développement professionnel en enseignement : approches théoriques. Revue des sciences de l'éducation, 31(1), 133-155.

VanTassel-Baska, J., Xuemei Feng, A., Brown, E., Bracken, B., Stambaugh, T., French, H., ... \& Bai, W. (2008). A study of differentiated instructional change over 3 years. Gifted Child Quarterly, 52(4), 297-312.

Warburton, D. E., \& Bredin, S. S. (2017). Health benefits of physical activity: a systematic review of current systematic reviews. Current opinion in cardiology, 32(5), 541-556. 